\title{
Determination of the Earth Fault Factor in Power Systems for Different Earthed Neutrals
}

\author{
M.R.A. Calado ${ }^{1}$, S.J.P.S. Mariano ${ }^{1}$ \\ ${ }^{1}$ Department of Electromechanical Engineering \\ University of Beira Interior \\ Calçada Fonte do Lameiro, 6201-001 Covilhã (Portugal) \\ Phone/Fax number:+351275329760/972, e-mail: rc@ubi.pt,sm@ubi.pt
}

\begin{abstract}
This paper presents an analytical method to calculate the earth fault factor (EFF) in power systems, under different neutral earthed possibilities. The magnitude of the EFF depends on the way the neutrals of a system are earthed and influences some important aspects in power systems as the sensitivity and selectivity of relays, the value of short-circuit currents, the overvoltage between lines and earth, the determination of arrester rating and the system insulation level. The obtained results are compared with the values of EFF referred in International Standards. The application of the proposed study is discussed.
\end{abstract}

\section{Keywords}

Earth Fault Factor, Fault location, Overvoltage, Grounding, Power systems.

\section{Introduction}

The grounding conditions, i.e., the neutral connection to earth, in power systems can be characterized by a factor called earth fault factor (EFF). There are a number of values, for any system, since there are many points of connection to earth, and therefore many other values for the equivalent parameters of the system. This factor, referred in both Portuguese and European Standards, determines the behaviour of the power system, influencing the value of short-circuit currents, the overvoltage between lines and earth [1], the determination of arrester rating and the system insulation level. The EFF, $k_{e}$, at a given location is the ratio of the root-mean-square value of the highest phase-to-earth voltage on a healthy phase during an earth fault, affecting one or more phases at one point on the system, to the root-mean-square value of the phase-to-earth voltage, which should be obtained at the given location in the absence of any such fault.

Depending on the topology and characteristics of the three-phase power systems, the principles and conditions for its determination must take into account the equivalent parameters of the circuit, the impedance down to the ground and the point where the defect occurs. Thus the achievement of this factor is not trivial and must be calculated for any location in the system.

The literature covering this issue is mainly focusing in the adoption of a value, which is used to chose the arresters rating [2], [3], calculate the system insulation level, and calculate the fault current for a system [4], [5], instead of accurately calculate the real EFF.

This paper proposes a methodology to calculate the EFF (the results are obtained using a developed software) and its values are compared with the values found in Standards, concluding about the application of this study to electrical power systems. To develop this analysis, a previous work concerning the adopted circuit and parameters in power systems was carried out and the different connections of neutral [6] to the earth were identified.

This paper is organized as follows. In Section 2 the considerations about power system networks and the influence of the EFF in their behaviour are presented. Section 3 describes the developed analytical method to the calculation of the EFF. In Section 4, a discussion in turn of the established and adopted EFF values is performed, and the main conclusions are presented in Section 5.

\section{The influence of the EFF on power systems}

It is well known that the system voltages to earth in a three-phase electrical system become unbalanced when one or more phases are grounded. The voltages of the healthy phases can assume values higher or smaller, depending on the system conditions in the instant of occurrence of the fault.

These voltages have a direct impact on the provision of the insulation system and the protective devices, and also in the neutral impedance to be used. For example, in the determination of arrester rating is very important the knowledge of the voltages to earth, as it must support that voltage without the possibility of compromise its protection task. This knowledge can be achieved through tests (not advisable) or by burdensome calculus. 
A procedure used in [7] turns easier the work to obtain the voltages to earth. This procedure is based on the adoption of estimation curves from the knowledge of the impedances of the system, using the Method of Symmetrical Components. This method only can be applied if the assumption that the positive and negative components of the impedances are equal is adopted. The obtained voltage only relates to the point of the fault, neglecting all the effects in the system. Depending on the type of the neutral connection to the earth, the voltages are obtained based on the symmetrical components of the impedance. For systems strictly grounded, the main relation is the ratio $\frac{X_{h}}{X_{d}}$, involving the zero and positive components of the impedance, being in this case a positive ratio. For isolated systems, the same ratio determines the voltages, but now adopting negative values.

Another analysis, based on pre-determined curves, can be carried out with respect to the short-circuit current in power systems and the voltages that appear in the case of occurring a fault, depending on the system impedances. Both obtained current and fault voltages can be seen as behaviour indexes, appealing to impedance components ratio.

All found references about this particular issue are fault based on curves of impedance, and are applied either in obtaining the short-circuit current or the fault voltage.

The operation of the electrical power depends on the factor of the ground fault, as it influences, among others, some of the electrical characteristics of the system.

The value depends on several conditions, such as the adoption of equivalent schemes and equipment of the system, the method of connecting the neutral to ground, the type of defect and the its resistance, the voltage at the time of the fault and finally, the point where the fault occurs. It is possible, for a system, the determination of a large number of values of EFF, since we calculate it for many other points of the system. For all these reasons, it is notoriously difficult to attribute a value to EFF at a point in the system, since there are many parameters that influence the factor and because the operating conditions are constantly changing. However, being a factor that generally characterizes a system and the conditions of its grounding, is very important to establish a method to calculate the EFF, for any point and for all operating condition of the network.

\section{Analytical Calculation of the EFF}

As previously defined, and if a single phase-to-earth (PE) fault occurs in phase A, the EFF $k_{e}$ can be calculated, respectively for phase $\mathrm{B}, k_{e B}$, and phase $\mathrm{C}, k_{e C}$, as:

$$
k_{e B}=\left|\frac{\overline{U_{B}}}{\bar{E}}\right| \text { and } k_{e C}=\left|\frac{\overline{U_{C}}}{\bar{E}}\right|
$$

with $\overline{U_{B}}$ e $\overline{U_{C}}$ the voltages of phases $\mathrm{B}$ and $\mathrm{C}$, and $\bar{E}$ the nominal voltage.
For a double phase-to-earth fault (PPE) (phases B and C for example), the EFF $k_{e 2}$ is defined as the ratio of the voltage to ground in healthy phase A to the voltage in the same phase when any fault occurs:

$$
k_{e 2}=\left|\frac{\overline{U_{A}}}{\bar{E}}\right|
$$

Due to convenience purposes, it can also be used the connection to earth factor (ECF) $k_{u}$ instead of EFF, that are related each other by:

$$
k_{e}=\sqrt{3} k_{u} .
$$

The analysis, and related calculus, to the determination of the EFF is similar to the one performed to the calculation of fault currents in a specific system. For both cases the issue is the achievement of voltages in healthy phases, when some kind of fault occurs. Thus, and for a given network, the adopted equivalent circuit, Fig. 1, doesn't takes into account any load [8], that is, the network is seen as infinite and the line voltages, under normal conditions, are the nominal ones (or, for security reasons, $10 \%$ above the nominal value).

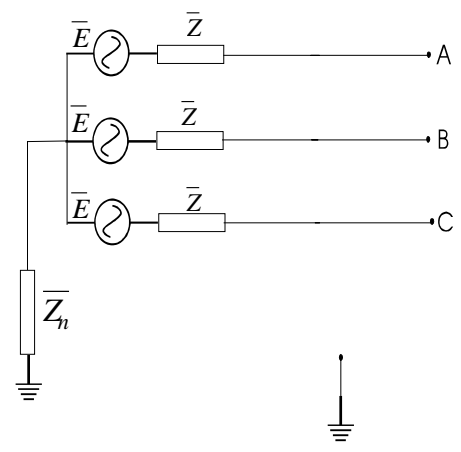

Fig. 1. System Equivalent circuit.

When a fault occurs, (PE or PPE), the system previously assumed to be symmetrical, becomes an asymmetric system, where it is impossible to perform calculations for a single phase, regardless the other phases. Thus, a method of analysis that simplifies the study with asymmetric voltages and currents systems must be adopted, as the Method of Symmetrical Components.

Considering a $\mathrm{PE}$ fault in phase $\mathrm{A}$, the voltages in phases $\mathrm{B}$ and $\mathrm{C}$ are, respectively [9]:

$$
\overline{U_{B}}=\frac{\left(a^{2}-a\right) \overline{Z_{i}}+\left(a^{2}-1\right) \overline{Z_{h}}+3 a \overline{Z_{Z}}}{\overline{Z_{d}}+\overline{Z_{i}}+\overline{Z_{h}}+3 \overline{Z_{Z}}} \overline{E_{1}}
$$

and

$$
\overline{U_{C}}=\frac{\left(a-a^{2}\right) \overline{Z_{i}}+(a-1) \overline{Z_{h}}+3 a \overline{Z_{Z}}}{\overline{Z_{d}}+\overline{Z_{i}}+\overline{Z_{h}}+3 \overline{Z_{Z}}} \overline{E_{1}}
$$

with 


$$
\begin{aligned}
& a=-\frac{1}{2}+j \frac{\sqrt{3}}{2} ; \\
& \overline{Z_{d}}=R_{d}+j X_{d} ; \\
& \overline{Z_{i}}=R_{i}+j X_{i} ; \\
& \overline{Z_{h}}=R_{h}+j X_{h} ;
\end{aligned}
$$

being $\overline{Z_{d}}, \overline{Z_{i}}$ and $\overline{Z_{h}}$ respectively the positive, negative and zero components of the impedance and the fault impedance is given by:

$$
\overline{Z_{Z}}=R
$$

Replacing (6) and (7) in (4) and (5), becomes:

$$
\left\{\begin{array}{l}
\overline{U_{B}}=\frac{\left(a^{2}-a\right)\left(R_{i}+j X_{i}\right)+\left(a^{2}-1\right)\left(R_{h}+j X_{h}\right)+3 a R}{R_{d}+j X_{d}+R_{i}+j X_{i}+R_{h}+j X_{h}+3 a R} \overline{E_{1}} \\
\overline{U_{C}}=\frac{\left(a-a^{2}\right)\left(R_{i}+j X_{i}\right)+(a-1)\left(R_{h}+j X_{h}\right)+3 a R}{R_{d}+j X_{d}+R_{i}+j X_{i}+R_{h}+j X_{h}+3 a R} \overline{E_{1}}
\end{array}\right.
$$

The equations in (8) show the complexity of the analysis of the EFF for any point of the system, and a good simplification it would be the parameterization related to the reactive parameter of the positive component of the impedance. Let be:

$$
\begin{aligned}
& x=\frac{X_{h}}{X_{d}} ; y=\frac{R_{h}}{X_{d}} ; \\
& c=\frac{R}{X_{d}} ; d=\frac{R_{d}}{X_{d}} ; r=\frac{X_{i}}{X_{d}} .
\end{aligned}
$$

Using (9), (8) becomes:

$$
\left\{\begin{array}{l}
\overline{U_{B}}=\frac{\left(a^{2}-a\right)(d+j r)+\left(a^{2}-1\right)(y+j x)+3 a c}{(2 d+y+3 a c)+j(1+r+x)} \overline{E_{1}} \\
\overline{U_{C}}=\frac{\left(a-a^{2}\right)(d+j r)+(a-1)(y+j x)+3 a c}{(2 d+y+3 a c)+j(1+r+x)} \overline{E_{1}}
\end{array}\right.
$$

Considering a PPE fault in phases B and C, the voltage in phase $\mathrm{A}$ is :

$$
\overline{U_{A}}=\frac{\overline{Z_{i}}\left[2\left(\overline{Z_{h}}+3 \overline{Z_{Z}}\right)+\overline{Z_{h}}\right]}{\overline{Z_{d}}\left(\overline{Z_{i}}+\overline{Z_{h}}+3 \overline{Z_{Z}}\right)+\overline{Z_{i}}\left(\overline{Z_{h}}+3 \overline{Z_{Z}}\right)} \overline{E_{1}}
$$

Once again, using (6) and (7) in (11), one obtain:

$$
\begin{aligned}
\overline{U_{A}} & =\frac{\left(R_{i}+j X_{i}\right)\left[2\left(\left(R_{h}+j X_{h}\right)+3 R\right)+\left(R_{h}+j X_{h}\right)\right]}{K} \bar{E}_{1} \\
& \text { with } \\
K & =\left(R_{d}+j X_{d}\right)\left(\left(R_{i}+j X_{i}\right)+\left(R_{h}+j X_{h}\right)+3 R\right)+ \\
& +\left(R_{i}+j X_{i}\right)\left(\left(R_{h}+j X_{h}\right)+3 R\right)
\end{aligned}
$$

Using (9), (12) is now:

$$
\begin{aligned}
\overline{U_{A}} & =\frac{[((y+j x)+3 c)+(y+j x)] \times 2(d+j r) \overline{E_{1}}}{K 1} \\
& \text { with } \\
K 1 & =(d+j 1)((d+j r)+(y+j x)+3 c)+ \\
& +(d+j r)((y+j x)+3 c)
\end{aligned}
$$

Presented methodology led to expressions that allow the calculation of the EFF, which depends on the parameters of the system. For convenience, were adopted the parameters $x, y, d, c$ and $r$.

Four Cases can be considered [10]. Let us describe the respective parameters of each of them:

$$
\text { - Case 1: } \begin{aligned}
& \overline{Z_{d}}=\overline{Z_{i}}=j X_{d} ; \\
& \overline{Z_{h}}=0 ; \\
& \overline{Z_{d}}=\overline{Z_{i}}=R_{d}+j X_{h} .
\end{aligned}
$$

- Case 2: $\overline{Z_{z}}=0$;

$$
\overline{Z_{h}}=R_{h}+j X_{h} \text {. }
$$

$$
\text { - Case 3: } \begin{aligned}
& \overline{Z_{d}}=j X_{d} ; \\
& \overline{Z_{i}}=j X_{i} ; \\
& \overline{Z_{z}}=0 ; \\
& \overline{Z_{h}}=R_{h}+j X_{h} .
\end{aligned}
$$

$$
\text { - Case 4: } \quad \begin{aligned}
& \overline{Z_{d}}=\overline{Z_{i}}=j X_{d} ; \\
& \overline{Z_{z}}=R ; \\
& Z_{h}=R_{h}+j X_{h} .
\end{aligned}
$$

All cases involve the reduced parameters $x$ and $y$. Cases 2, 3 and 4 also involve, respectively, the parameters $d, r$, and $c$.

In our analysis, we considered cases 1 and 3 . The first case was chosen because of impedance values that are adopted in most calculations of faults [11]. The third case was chosen as an example to study the influence of one of the other considered parameters. In this paper the obtained results for Case 1 are presented and discussed.

\section{A. EFF for Case 1}

Considering the parameters of the system for this case, equations (10) are now:

$$
\left\{\begin{array}{c}
\overline{U_{B}}=\frac{(-j \sqrt{3}) j X_{d}+\left(-1.5-j \frac{\sqrt{3}}{2}\right)\left(R_{h}+j X_{h}\right)}{R_{h}+j\left(2 X_{d}+X_{h}\right)} \overline{E_{1}} \\
\overline{U_{C}}=\frac{(j \sqrt{3}) j X_{d}+\left(-\frac{3}{2}+j \frac{\sqrt{3}}{2}\right)\left(R_{h}+j X_{h}\right)}{R_{h}+j\left(2 X_{d}+X_{h}\right)} \overline{E_{1}}
\end{array}\right.
$$


The voltage of phase $B$ as is stated in (14) can be introduced in (1) and (3), obtaining the ECF:

$$
k_{u B}=\left|\frac{\sqrt{3} X_{d}-1.5 R_{h}-1.5 j X_{h}-j \frac{\sqrt{3}}{2} R_{h}+\frac{\sqrt{3}}{2} X_{h}}{\left[j\left(2 X_{d}+X_{h}\right)+R_{h}\right] \sqrt{3}}\right|
$$

and, considering (9), $k_{u B}$ is:

$$
k_{u B}=\sqrt{\frac{\left(\sqrt{3}-\frac{3}{2} y+\frac{\sqrt{3}}{2} x\right)^{2}+\left(\frac{3}{2} x+\frac{\sqrt{3}}{2} y\right)^{2}}{3\left[(2+x)^{2}+y^{2}\right]}}
$$

or:

$$
\begin{aligned}
& \left(x^{2}+y^{2}\right)\left(3-3 k_{u B}^{2}\right)+x\left(3-12 k_{u B}^{2}\right)+ \\
& +y(-3 \sqrt{3})+\left(3-12 k_{u B}^{2}\right)=0
\end{aligned}
$$

dividing (17) by $\left(1-k_{u B}^{2}\right)$, one obtains:

$$
x^{2}+y^{2}+x\left(\frac{1-4 k_{u B}^{2}}{1-k_{u B}^{2}}\right)+y\left(-\frac{\sqrt{3}}{1-k_{u B}^{2}}\right)+\left(\frac{1-4 k_{u B}^{2}}{1-k_{u B}^{2}}\right)=0
$$

The equation (18) represents a circumference with centre in the point:

$$
\left(-\frac{1-4 k_{u B}^{2}}{2\left(1-k_{u B}^{2}\right)} ; \frac{\sqrt{3}}{2\left(1-k_{u B}^{2}\right)}\right)
$$

with radius:

$$
\sqrt{3} \frac{k_{u B}}{1-k_{u B}^{2}}
$$

Similarly, and for the healthy phase C:

$$
k_{u C}=\left|\frac{-\sqrt{3} X_{d}-1.5 R_{h}-j 1.5 X_{h}+j \frac{\sqrt{3}}{2} R_{h}-\frac{\sqrt{3}}{2} X_{h}}{\left[j\left(2 X_{d}+X_{h}\right)+R_{h}\right] \sqrt{3}}\right|
$$

and, considering (9), (21) becomes:

$$
k_{u C}=\sqrt{\frac{\left(-\sqrt{3}-\frac{3}{2} y-\frac{\sqrt{3}}{2} x\right)^{2}+\left(-\frac{3}{2} x+\frac{\sqrt{3}}{2} y\right)^{2}}{\sqrt{3} \times\left[(2+x)^{2}+y^{2}\right]}}
$$

that is:

$$
\left(x^{2}+y^{2}\right)\left(1-k_{u C}^{2}\right)+x\left(1-4 k_{u C}^{2}\right)+\sqrt{3} y+\left(1-4 k_{u C}^{2}\right)=0
$$

dividing by $\left(1-k_{u C}^{2}\right)$ :

$$
x^{2}+y^{2}+x\left(\frac{1-4 k_{u C}^{2}}{1-k_{u C}^{2}}\right)+y\left(\frac{\sqrt{3}}{1-k_{u C}^{2}}\right)+\left(\frac{1-4 k_{u C}^{2}}{1-k_{u C}^{2}}\right)=0
$$

Equation (24) describes a circumference with centre:

$$
\left(-\frac{1-4 k_{u C}^{2}}{2\left(1-k_{u C}^{2}\right)} ;-\frac{\sqrt{3}}{2\left(1-k_{u C}^{2}\right)}\right)
$$

and radius:

$$
\sqrt{3} \frac{k_{u C}}{1-k_{u C}^{2}}
$$

For a double phase-to-earth fault involving phases B and $\mathrm{C}$, and for the parameters of Case 1 , the voltage in phase $\mathrm{A}$ is given by:

$$
\overline{U_{A}}=\frac{3(y+j x)}{2 y+j(1+2 x)} \overline{E_{1}}
$$

Once more, appealing to (2) and (3), the ECF is:

$$
k_{u 2}=\frac{3}{\sqrt{3}} \sqrt{\frac{x^{2}+y^{2}}{4 x^{2}+4 y^{2}+4 x+1}}
$$

or:

$x^{2}\left(\frac{4}{3} k_{u 2}^{2}-1\right)+y^{2}\left(\frac{4}{3} k_{u 2}^{2}-1\right)+x\left(\frac{4}{3} k_{u 2}^{2}\right)+\left(\frac{1}{3} k_{u 2}^{2}\right)=0$

Dividing by $\left(4 / 3 k_{u 2}^{2}-1\right)$, one finally has the circumference given by:

$$
x^{2}+y^{2}+x\left(\frac{\frac{4}{3} k_{u 2}^{2}}{\frac{4}{3} k_{u 2}^{2}-1}\right)+\left(\frac{\frac{1}{3} k_{u 2}^{2}}{\frac{4}{3} k_{u 2}^{2}-1}\right)=0
$$

with centre:

$$
\left(-\frac{2 k_{u 2}^{2}}{2\left(4 k_{u 2}^{2}-3\right)} ; 0\right)
$$

and radius:

$$
\sqrt{3} \frac{k_{u 2}}{4 k_{u 2}^{2}-3}
$$

The characteristics obtained from this analysis, Fig. 2, represent areas for which the earth fault factor is constant.

The dashed lines represent segments of the circumference, which are obtained for different values of EFF in the healthy phase $\mathrm{B}$, when are considered different values of the parameters $x=\frac{X_{h}}{X_{d}}$ and $y=\frac{R_{h}}{X_{d}}$. The dotted circumferences represent the same, but for the healthy phase $\mathrm{C}$. 


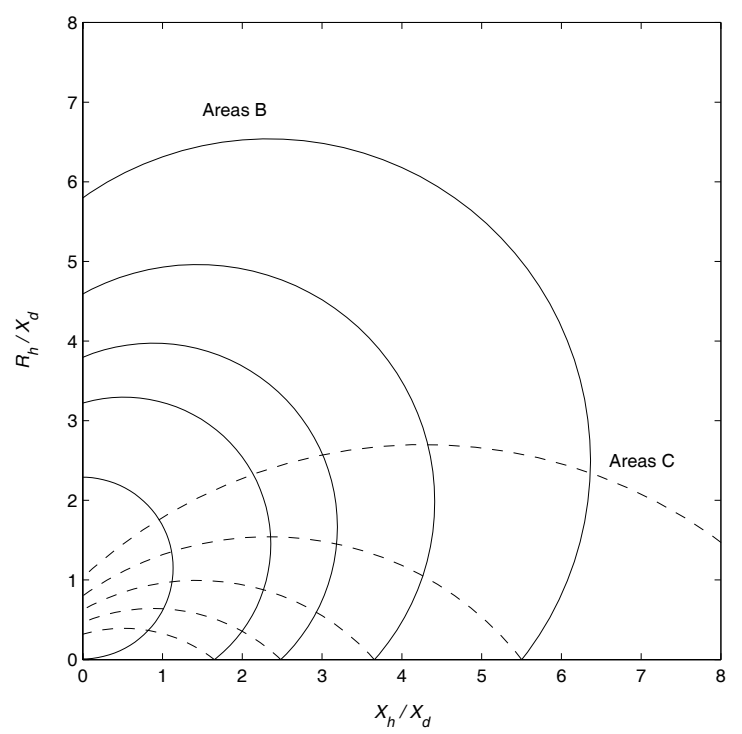

Fig. 2. Areas of constant EFF, for a PE fault.

The characteristics of Fig. 3 correspond to the results obtained for a double phase-to-earth fault in phases B and $\mathrm{C}$, thus represent the values of EFF for the healthy phase A.

Note that only the segments of the circumference that correspond to $x$ and $y$ positive are represented, because the system under study are solidly grounded, and in such a systems $\frac{X_{h}}{X_{d}}>0$.

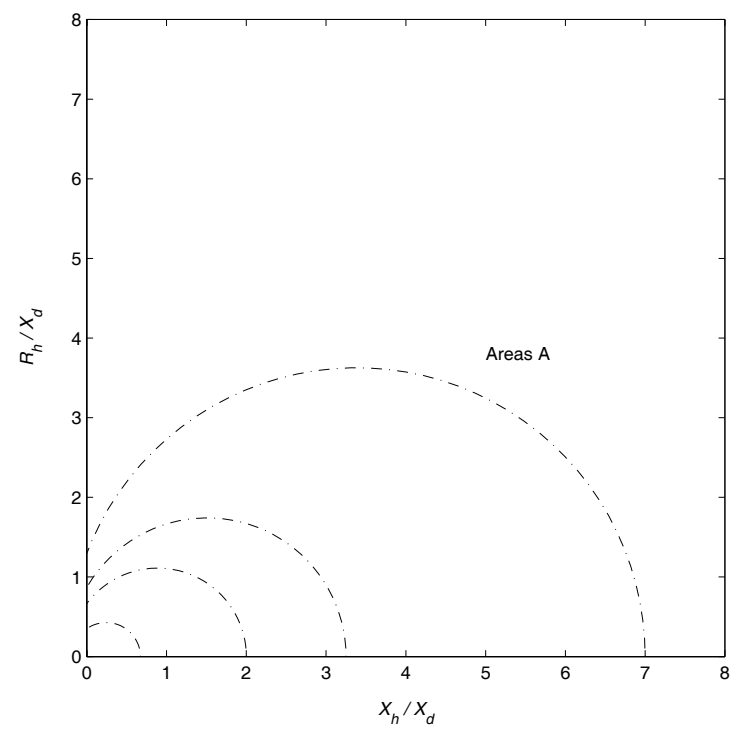

Fig. 3. Areas of constant EFF, for a PPE fault.

\section{EFF in Standards}

In Portuguese Standards NP 1853 [12], based on European Standards CEI 71, the EFF is referred, namely, is stated that for systems of high and very-high voltage the network must be connected to earth with $k_{e}$ less than 1.4 (for nominal voltages of $110 \mathrm{kV}$ ) or limited to 1.3 (for nominal voltages of $220 \mathrm{kV}$ ). According to that, such a values are guaranteed with manipulations in the ratios $\frac{R_{h}}{X_{d}}$ and $\frac{X_{h}}{X_{d}}$, as:

$$
\begin{aligned}
& \frac{R_{h}}{X_{d}} \leq 1 \text { and } \frac{X_{h}}{X_{d}} \leq 3 \text { for } k_{e} \leq 1.4 \\
& \text { and } \\
& \frac{R_{h}}{X_{d}} \leq 0.5 \text { and } \frac{X_{h}}{X_{d}} \leq 2 \text { for } k_{e} \leq 1.3
\end{aligned}
$$

Fig. 4 and Fig. 5 show the curves of $k_{e} \leq 1.3$, for Case 1 and Case 3, which analysis was developed previously for phases B and C, respectively.

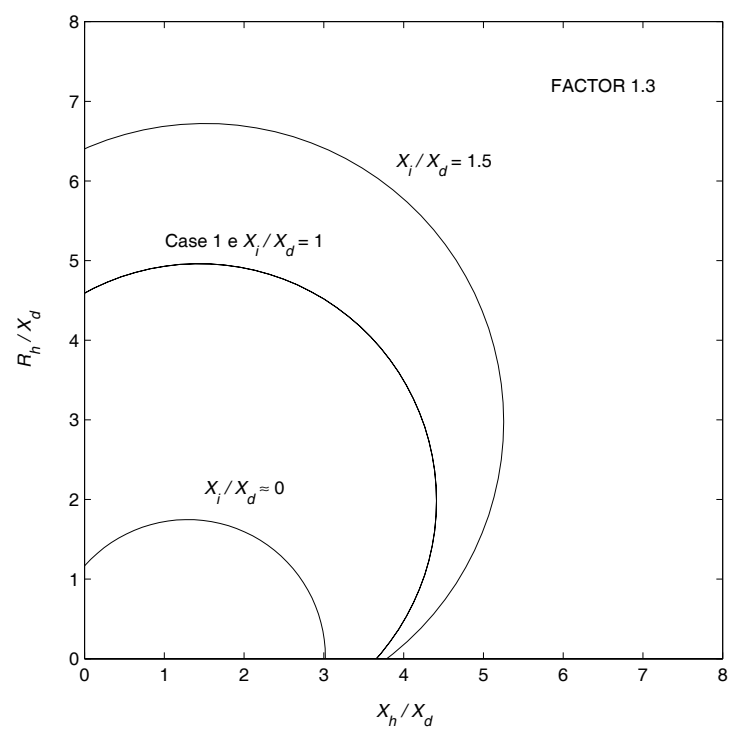

Fig. 4. Characteristics of EFF for phase B, limited by $k_{e}=1.3$.

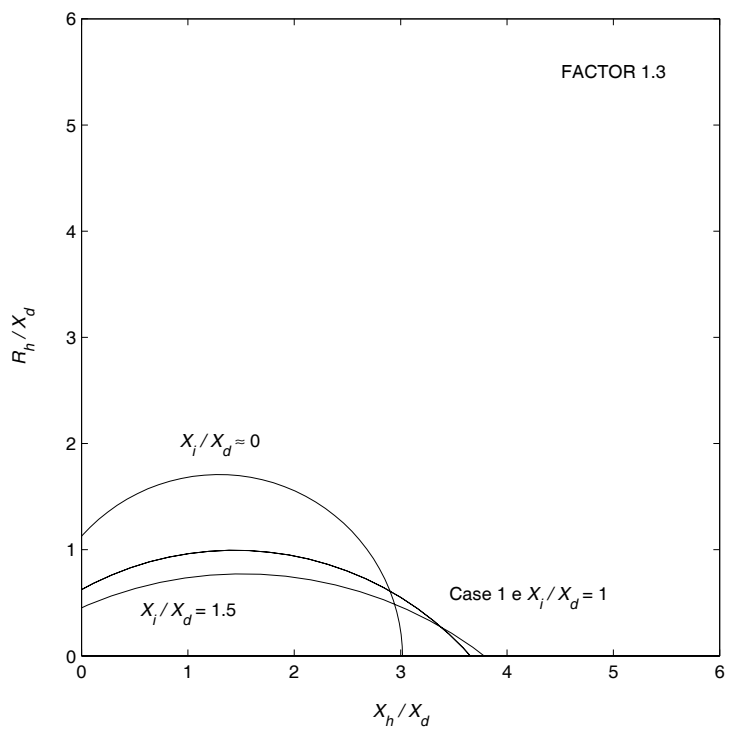

Fig. 5. Characteristics of EFF for phase C, limited by $k_{e}=1.3$.

Fig. 6 and Fig. 7 show the curves of $k_{e} \leq 1.4$, for Case 1 and Case 3 , and for phases $\mathrm{B}$ and $\mathrm{C}$, respectively. 
Comparing the curves shown here and the ratios $\frac{R_{h}}{X_{d}}$ and $\frac{X_{h}}{X_{d}}$ that lead to the referred EFF in standards, it was concluded that, for some cases, the limits of EFF are not possible to be guaranteed.

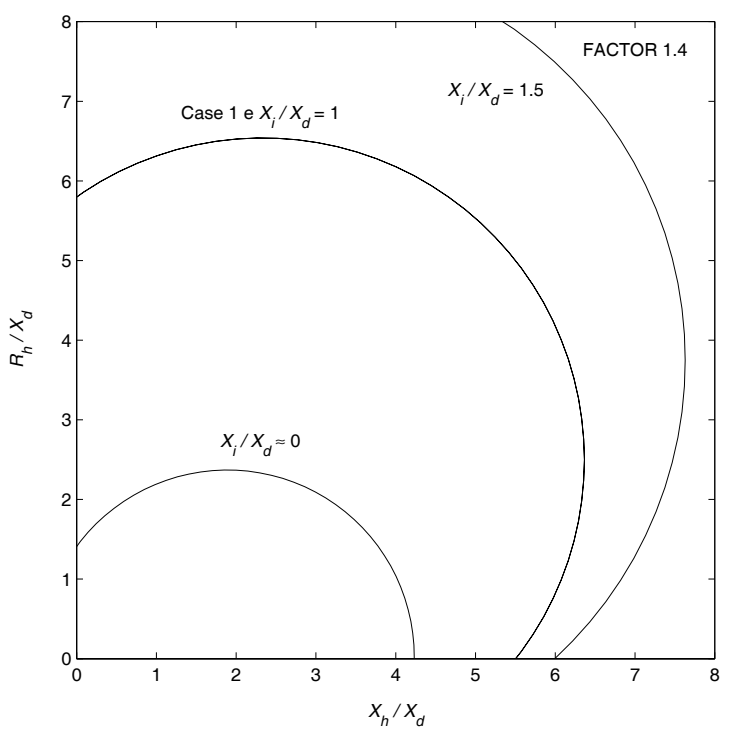

Fig. 6. Characteristics of EFF for phase B, limited by $k_{e}=1.4$.

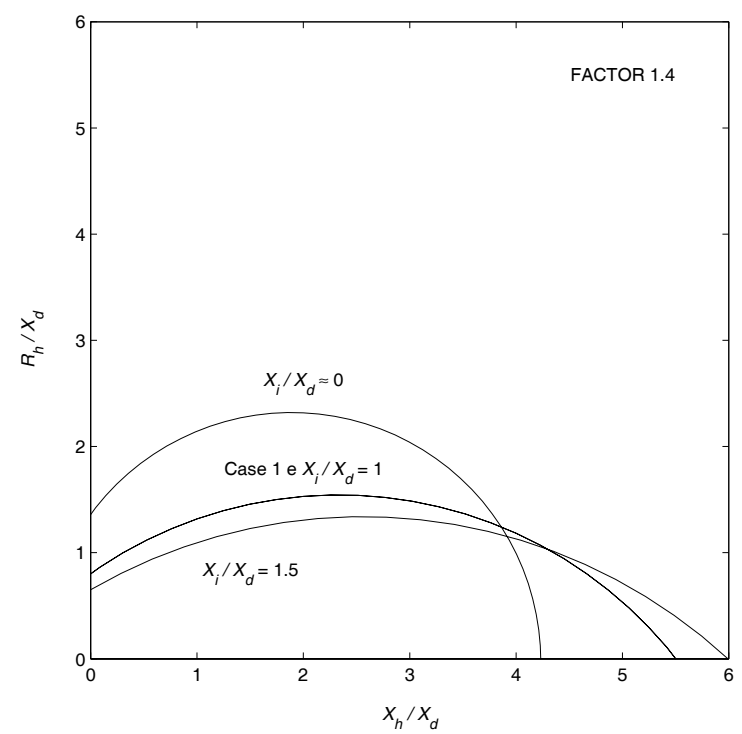

Fig. 7. Characteristics of EFF for phase C, limited by $k_{e}=1.4$.

\section{Conclusions}

The study here performed allows concluding that for constant values of the earth fault factor, and for different values of equivalent system parameters, it is possible to obtain families of circumferences. These circumferences are the boundaries of areas, which related with the values of $x=\frac{X_{h}}{X_{d}}$ and $y=\frac{R_{h}}{X_{d}}$, guarantee that the imposed value of EFF is not exceeded. Thus, one can, using the method of analysis developed here, make successive calculations and representations of curves, for any system and for any location of the ground fault, in order to regard the advisable values of EFF.

\section{References}

[1] Pirjo Heine and Matti Lehtonen, "Voltage Sag Distributions Caused by Power System Faults", IEEE Transactions on Power Systems, vol. 18, no. 4, pp. 13671373, November 2003.

[2] Jonathan Woodworth, Selecting Arrester MCOV and Uc. Part 1 of Arrester Selection Guide, Feb 12, 2009, Arresterorks 2009.

[3] Volker Hinrichse, Metal-Oxide Surge Arrester Fundamentals, Berlin, Germany: Siemens AG 2001.

[4] Wilfried Breuer and Norbert Christl, "Grid Access Solutions Interconnecting Large Bulk Power On- / Offshore Wind Park Installations to the Power Grid”, Proceedings of the Great Wall World Renewable Energy Forum GWREF 2006, October 23-27 2006, Beijing, China.

[5] S.Mohammad Shahrtash and Nabiollah Ramezani, "A Novel Method to Determine Earth Fault Split Factor For Grounding Grid Design of HV Substations”, Proceedings of the International Conference on Power System Technology POWERCON 2006, 22-26 October 2006, Chongqing, China.

[6] R. van de Sandt, J. Löwen, J. Paetzold and I. Erlich, "Neutral Earthing in Off-Shore Wind Farm Grids", Proceedings of the IEEE Bucharest Power Tech Conference, June 28th - July 2nd, 2009, Bucharest, Romania.

[7] E. M. Hunter, E. Pragst, P. H. Light, "Determination of Ground-Fault Current and Voltages on Transmission Systems-Part I", General Electric Review, SchenectadyN. Y., August 1939.

[8] Tom A., Electric power distribution equipment and systems, CRC Press, 2005.

[9] Jan Kamizierz Dobrogowski and Maria do Rosário Calado, "Alguns Aspectos da Ligação do Neutro à Terra nos Sistemas de Potência” (in Portuguese), Actas de las $5^{\text {as }}$ Jornadas Hispano-Lusas de Ingeniería Eléctrica, July 2 - 5, 1997, Salamanca, Spain.

[10] Jan Dobrogowski, "Earth Fault Factor Determination", Proceedings of the 6th. International Symposium on Short-Circuit Currents in Power Systems, Liège, September 1994.

[11] W. D. Stevenson, Jr., Elements of Power Systems Analysis, 4th. Edition, Mc. Graw-Hill International Book Company.

[12] DR III Série , No 246, NP 1853/1, 1853/2, 1853/3, (CEI 71/1, CEI 71/2, CEI 71/3, 1985) - Coordenação de Isolamento. 